\title{
A 9-year study of shigellosis in Northeast Malaysia: Antimicrobial susceptibility and shifting species dominance
}

\author{
Kirnpal-Kaur Banga Singh • Suvash Chandra Ojha • \\ Zakuan Zainy Deris • Rosliza Abdul Rahman
}

Received: 4 July 2010 /Accepted: 29 October 2010 /Published online: 19 November 2010

(C) The Author(s) 2010. This article is published with open access at Springerlink.com

\begin{abstract}
Aims In Malaysia, Shigella spp. is the third most common bacterial agent responsible for childhood diarrhoea. This study was conducted to determine the prevalence and antimicrobial susceptibility patterns of Shigella spp. isolated from patients admitted to the Hospital Universiti Sains Malaysia from January 2001 to December 2009.

Subjects and methods A hospital-based retrospective study was used. Stool samples from patients were cultured using a standard culture method. Shigella spp. isolates were identified by biochemical and serological methods, and the antimicrobial susceptibility pattern was evaluated using the Kirby-Bauer disc-diffusion method.

Results A total of 138 Shigella spp. were isolated from a total of 14,830 routine stool specimens, yielding an isolation rate of $0.93 \%$ that corresponded to $9.99 \%$ of the 1,381 bacterial pathogens isolated. Of these isolates, $S$. sonnei was the predominant species, followed by S. flexneri and S. boydii. Seasonal variation was noticed, and no significant differences were detected in the demographic data for S. flexneri and $S$. sonnei. The susceptibility of all isolated Shigella strains was tested against seven antibiotics. Ceftriaxone (99.1\%), ciprofloxacin (98.4\%), and nalidixic acid (93.8\%) were effective
\end{abstract}

Kirnpal-Kaur Banga Singh and Suvash Chandra Ojha contributed equally to this work.

K.-K. Banga Singh · S. C. Ojha · Z. Z. Deris · R. A. Rahman Department of Medical Microbiology and Parasitology,

School of Medical Sciences, Health Campus,

Universiti Sains Malaysia,

16150, Kubang Kerian, Kelantan, Malaysia

K.-K. Banga Singh $(\bowtie)$

Institute for Research in Molecular Medicine (INFORMM),

Health Campus, Universiti Sains Malaysia,

16150, Kubang Kerian, Kelantan, Malaysia

e-mail: kiren@kck.usm.my against the Shigella strains, whereas tetracycline and trimethoprim-sulfamethoxazole exhibited high frequencies of resistance (58.4\% and 53.8\%, respectively).

Conclusion This study is important for public health education aimed at reducing the morbidity and mortality associated with Shigella spp. infection. Our results also will be helpful for paediatricians and microbiologists in the selection of appropriate antibiotics for the management of diarrhoea.

Keywords Shigella Prevalence $\cdot$ Antimicrobial susceptibility $\cdot$ Diarrhoea

\section{Introduction}

Shigellosis, an intestinal infection caused by bacteria, is a global human health problem. It is one of the major epidemic diseases in the history of mankind, and it is one of the most common killing diseases of children all over the world. Shigellosis is the most important cause of bloody diarrhoea worldwide, especially in underdeveloped and developing nations with substandard hygiene and poor quality of water supplies; it also affects travelers from industrialised countries (Bennish and Wojtyniak 1991; Niyogi 2005). The four species of Shigella differ epidemiologically. S. dysenteriae is primarily associated with epidemics of serotype 1 and is associated with the highest fatality rate (5-15\%) (Ingersoll et al. 2002). S. flexneri predominates in areas of endemic infection and is the most frequently isolated species in developing countries (Faruque et al. 2002; Niyogi and Pazhani 2003; Zafar et al. 2005), and S. sonnei has been implicated in source outbreaks in developed countries (Katouli et al. 1989; Hale et al. 1991; Bonfiglio et al. 2002; Ekdaha and Andersson 2005; Hamamoto et al. 2000). S. boydii is 
associated with source outbreaks in Central and South America and is rarely isolated in North America (Kalluri et al. 2004). Kotloff et al. (1999) reported the percentage distribution of S. flexneri, S. sonnei, S. boydii, and $S$. dysenteriae to be $60 \%, 15 \%, 6 \%$, and $6 \%$, respectively, in developing countries, and $16 \%, 77 \%, 2 \%$, and $1 \%$, respectively, in developed countries. Outbreaks generally are associated with contaminated water, food, overcrowded communities, food handlers, and flies (Kapperud et al. 1995; Shears 1996).

Shigella infection occurs by the faecal-oral route. The minimum infective dose is extremely low; as few as 10 100 bacilli can initiate the disease, as it can survive gastric acidity better than other enterobacteria. The bacilli infect the epithelial cells of the villi in the large intestine and multiply inside them, spreading laterally to adjacent cells and penetrating into the lamina propria (Warren et al. 2006). Shigella infection can lead to illness ranging from mild, self-limited diarrhoea to severe dysentery with frequent passage of blood and mucus, high fever, abdominal pain, abdominal cramping, malaise, chills, nausea, vomiting, tenesmus, and, in rare cases, bacteraemia. Complications of shigellosis occur most frequently in children, the elderly, and immunocompromised patients. Prompt treatment with effective antimicrobial agents may shorten the duration of clinical symptoms and reduce the spread of infection (Replogle et al. 2000).

A definitive diagnosis of shigellosis can be made by isolating the organism from a stool sample. Shigellosis is one of the few enteric infections for which antimicrobials are prescribed. Shigella species have managed to survive the antibiotic era via an ingenious mechanism of resistance that has complicated the selection of empirical agents for its treatment (WHO 2001, 2005; Sivapalasingam et al. 2006; Kansakar et al. 2007; Kuo et al. 2008; Djie-Maletz et al. 2008; Sire et al. 2008). Moreover, antimicrobial resistance among Shigella spp. varies from region to region (Bhattacharya et al.
2005; Pazhani et al. 2005; Taneja 2007). Thus, when choosing an appropriate antibiotic to treat shigellosis, it is important to understand the local antimicrobial resistance. Herein, we report the prevalence of various Shigella species as well as the local antibiotic susceptibility pattern in Northeast Malaysia.

\section{Materials and methods}

Study design and subjects

A retrospective study was conducted by collecting data from the Department of Medical Microbiology and Parasitology, Hospital Universiti Sains Malaysia (HUSM), Malaysia. The institute, an 800-bed tertiary-care teaching hospital, provides low-cost medical care to the regional population of the Northeastern region of Malaysia as well as to the population of the border area of the neighboring country, Thailand. Stool specimens were collected from patients with clinically suspected dysentery who were admitted to HUSM between January 2001 and December 2009. Most of these patients visited HUSM with complaints of abdominal pain, vomiting, diarrhoea, and fever. Faecal specimens were collected from patients of all age groups. Demographic data such as age and sex, and the season during which the illness occurred were collected by questionnaire. These data were analysed using SPSS version 12 (SPSS Inc., USA). The association among Shigella spp., age, and sex was analysed using Fisher's exact test. The differences among the groups were considered to be statistically significant at $\mathrm{p}<0.05$.

Sample collection

During the study period, faecal samples were collected from patients in a clean, wide-mouth, sterile container. Freshly
Fig. 1 Distribution of Shigella isolates $(n=120)$ by age group from HUSM from 2001-2009

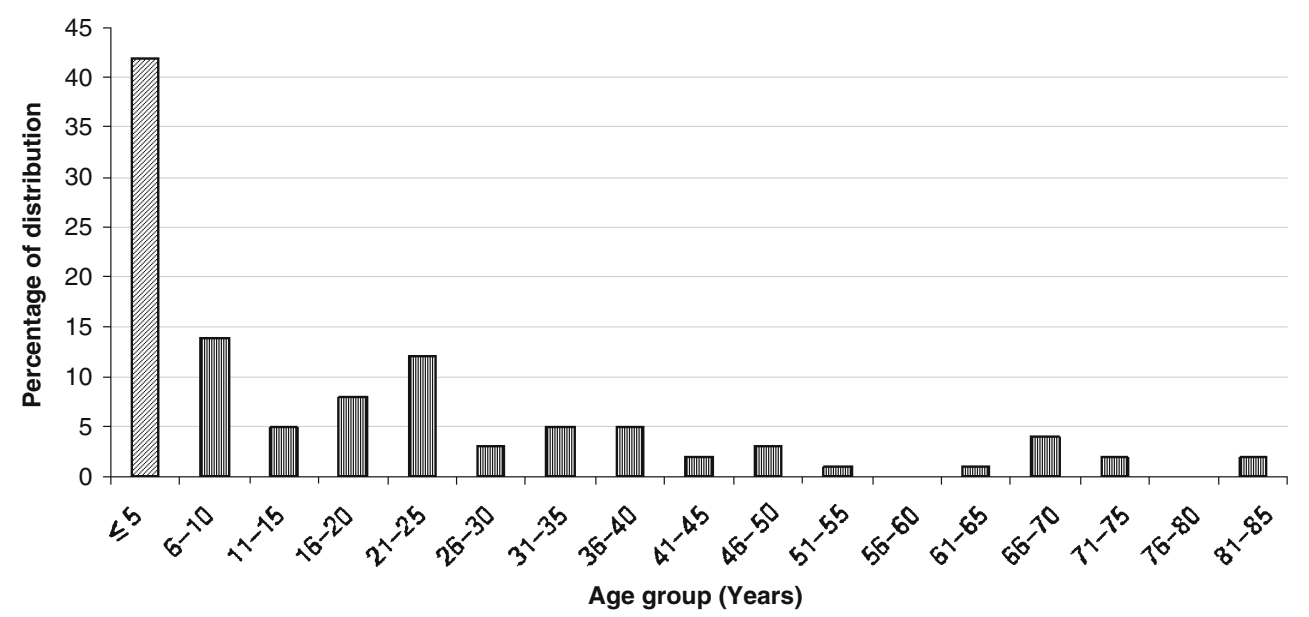


Table 1 Distribution of Shigella isolates by age group and gender

\begin{tabular}{lllll}
\hline & & S.flexneri & S. sonnei & P-value \\
\hline Age group $(\mathrm{n}=120)$ & Paediatrics & $\mathrm{n}=33(57.9 \%)$ & $\mathrm{n}=33(52.4 \%)$ & 0.585 \\
& Adult & $\mathrm{n}=24(42.1 \%)$ & $\mathrm{n}=30(47.6 \%)$ & \\
Gender $(\mathrm{n}=135)$ & Male & $\mathrm{n}=28(41.8 \%)$ & $\mathrm{n}=27(39.7 \%)$ & 0.862 \\
& Female & $\mathrm{n}=39(58.2 \%)$ & $\mathrm{n}=41(60.3 \%)$ & \\
\hline
\end{tabular}

collected faecal specimens were transported within $2 \mathrm{~h}$ of collection to the clinical laboratory for further processing.

\section{Bacteriological examination}

Stool specimens were plated on MacConkey and deoxycholate citrate agar (DCA) using a sterile inoculation loop. At the same time, another subsample was enriched in selenite $\mathrm{F}$ broth and incubated overnight at $37^{\circ} \mathrm{C}$. The next day, enriched broth was subcultured on MacConkey agar and DCA, and incubated overnight at $37^{\circ} \mathrm{C}$. Colonies morphologically resembling Shigella spp. were further evaluated with biochemical tests using triple sugar iron (TSI), urea agar slant, methyl red (MR), Simmon's citrate agar slant, and sulphur indole motility (SIM) media. Colonies were serologically confirmed by slide agglutination with appropriate group-specific polyvalent antisera, followed by type-specific monovalent antisera (DenkaSeikan, Tokyo, Japan). Non-serotypable isolates were further identified by API 20E (BioMerieux, France).

Drug susceptibility test

Antibiotic susceptibility of the Shigella isolates was tested using the Kirby-Bauer disc-diffusion method on MullerHinton agar plates following the guidelines of the Clinical and Laboratory Standards Institute (CLSI 2005). An isolate that is able to grow in the presence of an antibiotic in the medium is considered to be resistant. Resistance or sensitivity is defined according to the size of the zone of inhibition around the discs, and it correlates with the standard minimal inhibitory concentration (MIC), which is the lowest concentration that visibly inhibits bacterial growth. This correlation permits extrapolation from this simple test to the more complicated agar or broth dilution MIC test. A control strain of Escherichia coli (ATCC 25922) was included in the test as a quality control. Commercially manufactured discs (Oxoid, Hampshire, England) containing antimicrobial agents were used, and their concentrations in $\mu \mathrm{g} / \mathrm{ml}$ were as follows: ampicillin-10 (AMP), ciprofloxacin5 (CIP), trimethoprim-sulphamethoxazole-25 (SXT), nalidixic acid-30 (NAL), chloramphenicol-30 (CHL), ceftriaxone-30 (CRO), and tetracycline-30 (TCY). For NAL, only data for the years 2001, 2002, and 2006-2009 were included.

\section{Results}

\section{Bacteriological examination}

During the 9-year study period, a total of 14,830 routine stool samples were collected from patients with clinically suspected dysentery, and laboratory examinations of these samples were carried out to determine the prevalence of Shigella isolates. A total of 138 Shigella-specific strains were isolated from 1,381 isolates positive for bacterial pathogens, which corresponded to $9.99 \%$ of the total bacterial pathogens isolated. Shigella strains were isolated from specimens from symptomatic patients of all age groups. S. sonnei was the most prevalent isolate $(n=69$, $50.0 \%$ ), followed by $S$. flexneri $(\mathrm{n}=68,49.3 \%)$ and $S$.
Fig. 2 Distribution of Shigella isolates based on months (accumulation for the year 2001 to 2009). *The distribution of each Shigella species is expressed in percentage, and the total isolates are expressed in number

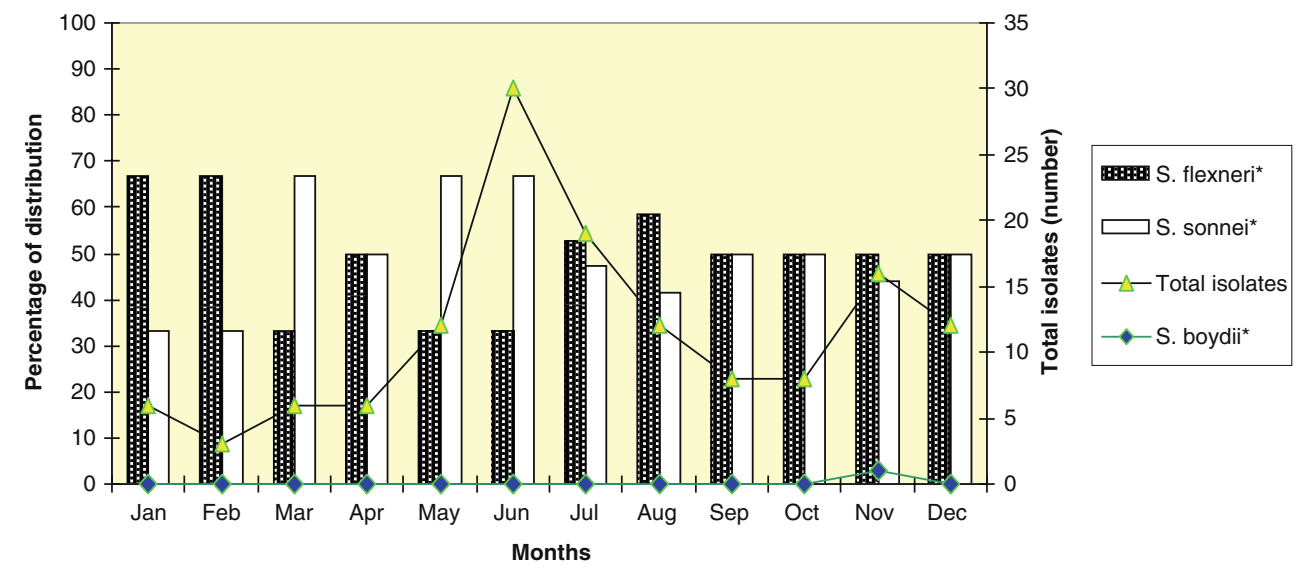


Fig. 3 Yearly distribution of Shigella isolates. *The distribution of each Shigella species is expressed in percentage, and the total isolates are expressed in number

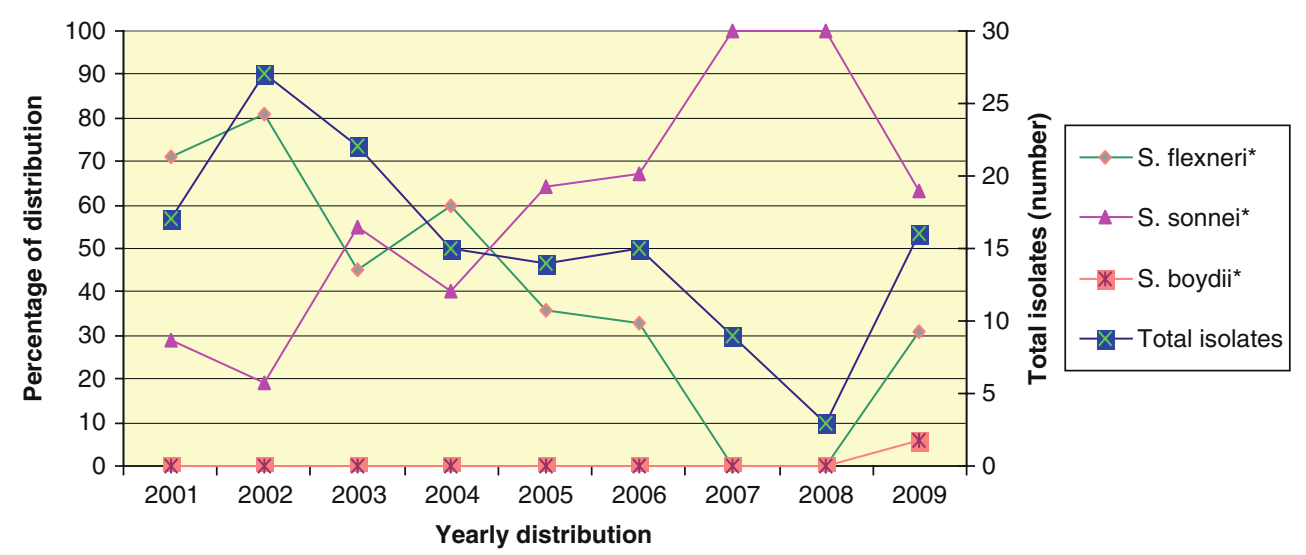

boydii $(\mathrm{n}=1,0.7 \%)$. Eighty-six Shigella strains $(61.8 \%)$ were isolated from paediatric patients younger than 15 years of age, and $42.0 \%$ of the total positive samples was obtained from children $\leq 5$ years old. Figure 1 shows the distribution of Shigella isolates by age group in 5-year age intervals. In this study, demographic data for S. flexneri and $S$. sonnei were not significantly different (Table 1).

Seasonality and yearly distribution

In Malaysia, environmental conditions remain almost the same throughout the year, with the exception of the rainy season in November and December. Figure 2 shows that the seasonal distribution of infection with Shigella spp. Shigellosis occurred throughout the year, but was more prevalent during the months of May to August, followed by the rainy season.

Figure 3 shows the yearly distribution of the Shigella isolates. S. flexneri was the most common species isolated in 2001 (70.6\%), 2002 (81.5\%), and 2004 (60.0\%). In 2003, a shift occurred, and S. sonnei replaced S. flexneri as the most prevalent species. Subsequently, a gradual decreasing trend for S. flexneri was observed until 2008. In 2009, S. sonnei remained the dominant species, although a few strains of $S$. flexneri were isolated.
Drug susceptibility

Figure 4 shows the results of the tests of antimicrobial susceptibility of Shigella isolates against seven antimicrobial agents. The highest sensitivity was detected for CRO (99.1\%), followed by CIP (98.4\%) and NAL (93.8\%). High sensitivity of Shigella isolates to CHL (68.0\%) and AMP $(67.4 \%)$ also was detected. The most frequent antibiotic resistance was observed for TCY and SXT, with resistance rates of $58.4 \%$ and $53.8 \%$, respectively. Resistance to one or more drugs was observed in $100 \%$ of the 138 isolates. The frequency of resistance for TCY, SXT, AMP, and CHL was the highest compared to the other antibiotics.

A comparative analysis of the sensitivity of the Shigella isolates against the individual antibiotics was performed. The highest sensitivity of $S$. sonnei occurred with CRO (98.4\%), followed by CHL (96.9\%), CIP (96.8\%), AMP (95.5\%), and NAL (95.2\%). The resistance percentage for S. sonnei was greatest for TCY (52.7\%), followed by SXT (52.9\%). S. flexneri was most sensitive to CIP (100\%) and CRO (100\%), followed by NAL (96.2\%). S. flexneri was most resistant to TCY (73.3\%), SXT (54.0\%), CHL (66.0\%), and AMP (60.0\%). S. boydii showed the highest sensitivity to CIP, CRO, and NAL, and was resistant to the remaining antimicrobial agents tested.
Fig. 4 Antibiotics sensitivity pattern of Shigella isolates. AMP (ampicillin); CIP (ciprofloxacin); SXT (trimethoprim-sulfamethoxazole); CHL (chloramphenicol); CRO (ceftriaxone); TCY (tetracycline)

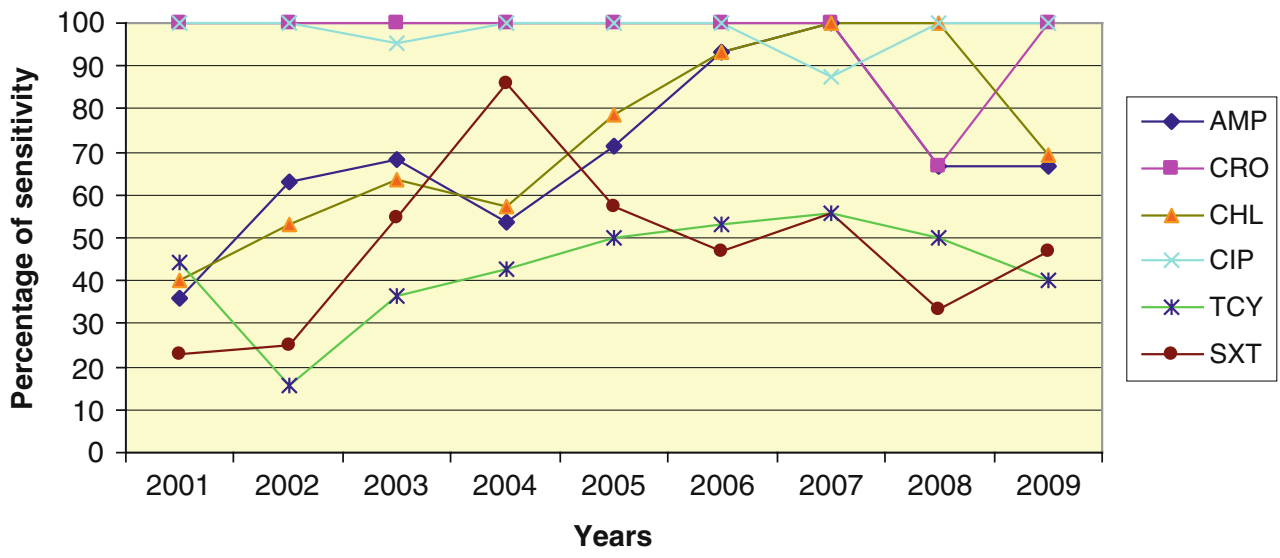




\section{Discussion}

Shigellosis is the most communicable of the bacterial diarrhoeas. According to the WHO, an estimated 165 million episodes of shigellosis occur annually, resulting in 1.1 million fatalities (Kotloff et al. 1999). Because shigellosis is highly contagious, awareness of the prevalence of the disease, the identity of the dominant strain, and the antimicrobial susceptibility of the dominant strain is crucial to ensuring proper clinical treatment and patient management, and for epidemiological investigation purposes.

Shigella spp. still accounts for a significant proportion of bacillary dysentery in many tropical and subtropical countries. In the Lee and Puthacheary (2003) study of bacterial enteropathogens in a Malaysian urban hospital, Shigella spp. were isolated from 1.4\% (386 isolates) of total stool specimens, corresponding to $13 \%$ of the 2,986 isolates positive for bacterial pathogens. In our study the isolation rate was $0.93 \%$, corresponding to $9.99 \%$ of the 1,381 isolates positive for bacterial pathogens. The possible underestimation of Shigella spp. prevalence can result from both delayed transportation and non-use of a transport medium. Over the course of the 9 -year study, the isolation rate did not vary for S. sonnei (50.0\%) and S. flexneri (49.3\%), and S. dysenteriae was not detected at all.

The monthly distribution of Shigella spp. did not play an important role in shigellosis prevalence. The infection occurred throughout the year, with the highest isolation in June followed by a second peak in November (i.e., two peaks each year). In 2001, 2002, and 2004, S. flexneri was the most common of the four species, whereas in 2003 and from 2005 to 2009 , S. sonnei was the dominant species. This finding differs from those of studies conducted in other developing countries (Faruque et al. 2002; Niyogi and Pazhani 2003; Zafar et al. 2005), where S. flexneri was the most frequently isolated species; however, our results match findings in developed countries (Katouli et al. 1989; Hale et al. 1991; Hamamoto et al. 2000; Bonfiglio et al. 2002; Ekdaha and Andersson 2005).

In our study, Shigella spp. were isolated from stool specimens of symptomatic patients of all age groups (from $<1$ month to $>85$ years of age) seen in our hospital. Of these, the majority of Shigella-specific strains were obtained from paediatric patients younger than 15 years of age, and the highest prevalence was seen in the $\leq 5$ years age group. This finding was in agreement with the results of Lee and Puthacheary's (2002) study of patients with diarrhoea admitted to the University of Malaya Medical Centre (UMMC), Kuala Lumpur, Malaysia. Their study showed that diarrhoea due to Shigella is a disease that affects all ages, but that it is commonly seen in paediatric patients. Other studies also have reported that the highest prevalence of Shigella is seen in the $\leq 5$ years age group (Arya et al. 1977; Mutanda et al. 1979; MoezArdalan et al. 2003).

Shigella spp. were most sensitive to the antibiotics CRO, CIP, and NAL, and the sensitivity did not vary much among species or strains; this finding is similar to the results reported by Fulla et al. (2005). Resistance to individual drugs varied among the three Shigella species, and this was especially true for AMP and CHL. All three Shigella species also exhibited a very high rate of resistance to TCY and SXT. A similar pattern of resistance has been reported in Pakistan, Turkey, India, and Nepal (Ayser and Guriz 1998; Fulla et al. 2005; Pazhani et al. 2005). We found significant differences in the antimicrobial susceptibility of $S$. flexneri and $S$. sonnei to AMP and CHL. Overall, S. flexneri was more frequently resistant to TCY, SXT, AMP, and CHL, alone or in combination, than was S. sonnei. A possible explanation is that infections due to $S$. sonnei are clinically less severe than infections due to the other Shigella species, making exposure to selective pressure from antibiotics less likely.

These results strongly suggest that TCY and SXT can no longer be used to treat cases of severe diarrhoea and dysentery at HUSM. Although our sample size may not reflect the genuine spectrum of Shigella spp. in this part og Malaysia, our results are significant: To our knowledge, this study is the first to describe the prevalence of Shigella spp. and their antimicrobial resistance patterns in Northeastern Malaysia.

In conclusion, our study demonstrates that $S$. sonnei is currently the predominant species at HUSM. The antimicrobial resistance pattern suggests widespread resistance of Shigella to TCY and SXT. However, recommendations about antimicrobial treatment must be updated regularly based on surveillance results.

Acknowledgements We gratefully acknowledge the Institute for Postgraduate Studies, Universiti Sains Malaysia, for providing fellowship assistance and the staff of the Department of Medical Microbiology and Parasitology, Hospital Universiti Sains Malaysia (HUSM), Kelantan, Malaysia, for assistance with sample processing.

Conflict of interest All of the authors contributed to, read, and approved the final, submitted version of the manuscript, and they do not have any conflict of interest.

Open Access This article is distributed under the terms of the Creative Commons Attribution Noncommercial License which permits any noncommercial use, distribution, and reproduction in any medium, provided the original author(s) and source are credited. 


\section{References}

Arya D, Chikara NL, Agarwal KC, Ganguli NK (1977) Shigellosis in Chandigarh. Ind J Pathol Microbiol 20:15-21

Ayser AD, Guriz H (1998) Drug resistance of Shigella strains isolated in Ankara, Turkey 1993-1996. Scand J Infec Dis 30:351-353

Bennish ML, Wojtyniak BJ (1991) Mortality due to shigellosis: community and hospital data. Rev Infect Dis 13:S245-S251

Bhattacharya S, Khanal B, Bhattaarai NR, Das ML (2005) Prevalence of Shigella species and their antimicrobial resistance patterns in eastern Nepal. J Health Popul Nutr 23:339-342

Bonfiglio G, Simpore J, Pignatelli S, Musumeci S, Solinas ML (2002) Epidemiology of bacterial resistance in gastro-intestinal pathogens in a tropical area. Int J Antimicrob Agents 20:387-389

Clinical and Laboratory Standards Institute (2005) Performance standards for antimicrobial susceptibility testing: fifteenth informational supplement. CLSI/NCCLS document M100-S15. Clinical and Laboratory Standards Institute, Wayne, $\mathrm{Pa}$

Djie-Maletz A, Reither K, Danour S, Anyidoho L, Saad E, Danikuu F (2008) High rate of resistance to locally used antibiotics among enteric bacteria from children in Northern Ghana. J Antimicrob Chemother 61:1315-1318

Ekdaha K, Andersson Y (2005) The epidemiology of travel-associated shigellosis-regional risks, seasonality and serogroups. J Infect 51:322-329

Faruque SM, Khan R, Kamruzzaman M, Yamasaki S, Ahmad QS, Azim T, Nair GB, Takeda Y, Sack DA (2002) Isolation of $S$. dysenteriae type 1 and $S$. flexneri strains from surface waters in Bangladesh: Comparative molecular analysis of environmental Shigella isolates versus clinical strains. Appl Environ Microbiol 68:3908-3913

Fulla N, Prado V, Dur NC, Lagos R, Levine M (2005) Surveillance for antimicrobial resistance profile among Shigella species isolated from a semirural community in the northern administrative area of Santiago, Chile. Am J Trop Med Hyg 72:851-854

Hale TL, Keush GT, Formal SB (1991) Shigella. In Medical Microbiology (Baron, S., ed). Churchill Livingstone, US

Hamamoto A , Kinosita K, Suyama N, Ishii Y, Kohno S (2000) A Shigella sonnei outbreak in Nagasaki. Kansenshogaku Zasshi 74:1004-1011

Ingersoll M, Groisman EA, Zychlinsky A (2002) Pathogenicity islands of Shigella. Curr Topics Microbiol Immunol 264:49-65

Kalluri P, Cummings KC, Abbott S, Malcolm GB, Hutcheson K, Beall A, Joyce K, Polyak C, Woodward D, Caldeira R, Rodgers F, Mintz ED, Strockbine N (2004) Epidemiologic features of a newly described serotype of Shigella boydii. Epidemiol Infect 132:579-583

Kansakar P, Malla S, Ghimire GR (2007) Shigella isolates of Nepal: changes in the incidence of Shigella subgroups and trends of antimicrobial susceptibility pattern. Kathmandu Univ Med J (KUMJ) 5:32-37

Kapperud G, Rorvik LM, Hasseltvedt V, Hoiby EA, Iversen BG, Staveland K, Johnson G, Leitao J, Heriksad H, Andersson Y (1995) Outbreak of Shigella sonnei infection traced to imported iceberg lettuce. J Clin Microbiol 33:609-614

Katouli M, Pachenary A, Jaafari A, Asghar A, Moghaddam F, Dehaghi NH (1989) The role of Shigella spp., in childhood diarrhoea in Iran and their antibiotic resistance. Scand. J Infect Dis 21:415-419

Kotloff KL, Winickoff JP, Ivanoff B, Clemens JD, Swerdlow DL, Sansonetti PJ, Adak GK, Levine MM (1999) Global burden of
Shigella infections: implications for vaccine development and implementation of control strategies. Bull World Health Organ 77:651-666

Kuo CY, Su LH, Perera J, Carlos C, Tan BH, Kumarasinghe G (2008) Antimicrobial susceptibility of Shigella isolates in eight Asian countries, 2001-2004. J Microbiol Immunol Infect 41:107-111

Lee WS, Puthacheary SD (2002) Bacterial enteropathogens isolated in childhood diarrhoea in Kuala Lumpur: the changing trend. Med J Malaysia 57:1-2

Lee WS, Puthacheary SD (2003) Species distribution and antibiotic resistance of Shigella isolated in an urban community in Malaysia. Med J Malaysia 58:262-267

MoezArdalan K, Zali MR, Dallal MM, Hemami MR, Salmanzadeh Ahrabi S (2003) Prevalence and pattern of antimicrobial resistance of Shigella species among patients with acute diarrhoea in Karaj, Tehran, Iran. J Health Popul Nutr 21:96-102

Mutanda LN, Kavita JN, Wamola IA (1979) Patterns of Shigella species and serotypes in East Africa. East Afr Med J 56:381-384

Niyogi SK (2005) Shigellosis. J Microbiol 43:133-143

Niyogi SK, Pazhani GP (2003) Multiresistant Shigella species isolated from childhood diarrhoea cases in Kolkata, India. Jpn J Infect Dis 56:33-34

Pazhani GP, Ramamurthy T, Mitra U, Bhattacharya SK, Niyogi SK (2005) Species diversity and antimicrobial resistance of Shigella species isolated between 2001 and 2004 from hospitalized children with diarrhoea in Kolkata (Calcutta) India. Epidemiol Infect 133:1089-1095

Replogle ML, Fleming DW, Cieslak PR (2000) Emergence of antimicrobial-resistant shigellosis in Oregon. Clin Infect Dis 30:515-519

Shears (1996) Shigella Infections. Ann Trop Med Parasitol 90:105-114

Sire JM, Macondo EA, Perrier-Gros-Claude JD, Siby T, Bahsoun I, Seck A (2008) Antimicrobial resistance in Shigella species isolated in Dakar, Senegal (2004-2006). Jpn J Infect Dis 61:307-309

Sivapalasingam S, Nelson JM, Joyce, Hoekstra M, Angulo FJ, Mintz ED (2006) High prevalence of antimicrobial resistance among Shigella isolates in the United States tested by the National Antimicrobial Resistance Monitoring System from 1999 to 2002. Antimicrob Agents Chemother 50:49-54

Taneja N (2007) Changing epidemiology of shigellosis and emergence of ciprofloxacin-resistant Shigella in India. J Clin Microbiol 45:678-679

Warren BR, Parish ME, Schneider KR (2006) Shigella as a food borne pathogen and current methods for detection in food. Crit Rev Food Sci Nutr 46:551-567

World Health Organization. Global strategy for containment of antimicrobial resistance. WHO/CDS/CSR/DRS/2001.2a. Geneva: WHO; 2001. Available at: http://www.who.int/drugresistance/WHO $\% 20$ Global $\% 20$ Strategy $\% 20 \% 20$ Executive $\% 20$ Summary\%20-\%20English\%20version.pdf (accessed December 2008)

World Health Organization. Guidelines for the control of shigellosis, including epidemics due to Shigella dysenteriae type 1 . Geneva: WHO; 2005. Available at: http://www.who.int/child adolescent_health/documents/9241592330/en/. (accessed December $20 \overline{0} 8$ )

Zafar A, Sabir N, Bhutta ZA (2005) Frequency of isolation of Shigella serogroups/scrotypes and their antimicrobial susceptibility pattern in children from slum area in Karachi. J Pak Med Assoc 55:184-188 